\title{
Editorial
}

Psychotherapy

and Psychosomatics

\section{Cyberchondria: Challenges of Problematic Online Searches for Health-Related Information}

\author{
Vladan Starcevic \\ Sydney Medical School, University of Sydney, and Department of Psychiatry, Nepean Hospital, Sydney, NSW, Australia
}

The digital revolution has changed many aspects of life, for example, the way in which health-related information is obtained. Such information is now very easy to access via the Internet, at little or no cost. For most people, the Internet has become the first stop when they need to find out more about symptoms, health and disease. The consequences of this change are being increasingly appreciated, but they are still poorly understood. While some people feel empowered by an easy access to health information and less anxious after seeking health information on the Internet, others are more anxious or puzzled [1]. This is likely to affect health care-seeking behaviour and relationship with physicians.

\section{What Is Cyberchondria?}

The concept of cyberchondria has been invoked to refer to the anxiety-amplifying effects of online health-related searches. Considering the origin of the term (i.e., as the counterpart of hypochondria in the digital age), cyberchondria refers to an abnormal behavioural pattern and emotional state. In other words, cyberchondria is not just a tendency to seek health-related information on the Internet, which has become a normal part of modern life. Several definitions of cyberchondria have been proposed and they all include excessive or repeated online searches

\section{KARGER}

(C) 2017 S. Karger AG, Basel

E-Mail karger@karger.com

www.karger.com/pps for health-related information and anxiety about health [2]. The direction of causality in the relationship between online health-related searches and health anxiety may vary from one individual to another. In some cases, perhaps a majority, a heightened health anxiety is primary and attempt to alleviate it is the reason for online healthrelated searches; however, these searches only cause more distress and make health anxiety worse [3-7]. In others who develop elevated levels of health anxiety after online health-related searches, there is no history of heightened health anxiety or hypochondriasis and their motivation for performing searches may be different (e.g., curiosity or appearance of a new symptom) [8-10]. It is important to note that regardless of what occurs first (health anxiety or online health-related searches), online health-related searches in cyberchondria result in higher levels of health anxiety compared to the levels of health anxiety prior to initiating these searches.

Taking into account these considerations, it can be proposed that cyberchondria denotes repeated online searches for health-related information that are associated with increasing levels of health anxiety. Health anxiety is conceptualised here in accordance with the revised version of the Diagnostic Criteria for Psychosomatic Research (DCPR) as a "generic worry about illness" [11]. This definition of cyberchondria primarily refers to the specific behaviour - online health-related searches; it

Vladan Starcevic

Department of Psychiatry, Nepean Hospital PO Box 63

Penrith, NSW 2751 (Australia)

E-Mail vladan.starcevic@sydney.edu.au 
does not suggest that cyberchondria is a distinct condition, much less an independent diagnostic entity. Also, it would be misleading to conceptualise cyberchondria solely as a modern "version" of hypochondriasis, a condition that contrary to DSM-5, continues to be recognised as a distinct disorder by the revised version of the DCPR [11]. While high levels of health anxiety have been associated with more Internet use to search for health-related information $[4,5,12,13]$, whereby online health-related searches usually serve the purpose of reassurance seeking or checking in the context of pre-existing hypochondriasis, people without pre-existing hypochondriasis may also become excessively anxious about their health as a result of these searches, as noted above. What appears to be shared by all individuals with cyberchondria is excessive amount of time spent performing the searches. This is usually at the expense of participating in other activities, which can have further negative consequences.

The Cyberchondria Severity Scale [7] was developed to measure the severity of cyberchondria. This instrument conceptualises cyberchondria as a multidimensional construct consisting of compulsion (unwanted nature of online health-related searches), distress (negative emotional states and physiological reactions associated with online health-related searches), excessiveness, reassurance (seeking reassurance from a medical professional) and mistrust of medical professionals (conflict as to whether one should trust one's own doctor or results of online searches).

\section{What May Be Specific for Cyberchondria?}

If the proposed definition of cyberchondria is correct, it is crucial to understand both how online health-related searches increase health anxiety and why this behavioural pattern continues despite its adverse effects. In this regard, the factors that are associated with hypochondriasis ("associated factors"), some of which may also play a role in cyberchondria, need to be distinguished from the factors that amplify health anxiety during online searches ("anxiety-amplifying factors"). This is because the latter may be specific for cyberchondria.

Among others, the associated factors include intolerance of uncertainty [14], need for thorough, "perfect" explanations of symptoms and other health complaints [15, 16] and selective attention to and perception of healthrelated information $[17,18]$. These factors may also act to maintain cyberchondria, thereby explaining the repetitiveness of online health-related searches. For example, a sense of uncertainty about one's health may increase during online health-related searches, especially when online information is ambiguous or conflicting; a need to quell that uncertainty and arrive at a "closure" may then drive further online searches. Likewise, if the Internet is perceived as the best medium to provide a "perfect" explanation for any health-related query because it contains information on "everything," this may fuel further online searches. Selective attention paid to health-related information found online may contribute to an increase in health anxiety and motivate some people to keep coming back to the Internet to reduce anxiety by continuing the searches.

Anxiety-amplifying factors pertain to various aspects of the online environment in which health-related searches take place. In such an environment, it may be more difficult to distinguish between credible and non-credible sources of information than in an offline, "real" world. Because of this, information obtained via unregulated (potentially less trustworthy) websites may be treated in the same way as information found on the "official" (potentially more trustworthy) websites, producing confusion and anxiety if there is a discrepancy in their content [19]. Furthermore, the Internet is not designed to always provide relevant, accurate, non-conflicting, non-ambiguous or reassuring information, which makes the outcome of online health-related searches less predictable compared to the outcome of seeking reassurance from a family member or physician [6]. Not only can the Internet provide conflicting, ambiguous or wrong information on the subject of the search, but it can be distracting and lead to other, potentially anxiety-amplifying information (e.g., information about hitherto unknown diseases or health risks). Some people may not realise that the Internet is not an ideal place to seek reassurance, with a failure to obtain the expected reassurance in the course of online health-related searches increasing their health anxiety.

Other anxiety-amplifying factors boil down to various difficulties with managing online information, especially online health information. It has been suggested that health anxiety may increase because of the erroneous beliefs about the ranking of search results, that is, a tendency to equate higher rankings of online search results with higher probabilities of providing an explanation [3]. Thus, an interpretation of the higher ranking of some malignancy as an explanation for the symptom that initiated an online search is very likely to increase health anxiety. Another anxiety-amplifying factor may be a difficulty managing abundant online information about health, especially if it is conflicting or vague $[6,9]$. Information 
overload is known to generate distress and anxiety [20] and some individuals who embark on an online healthrelated search are surprised to find out that "more" does not necessarily mean "better," as they do not expect abundance of information to increase their health anxiety.

Similar to the associated factors, anxiety-amplifying factors can also play a role in maintaining cyberchondria. Thus, difficulties in distinguishing between credible and non-credible sources of online information, expectations that the Internet will ultimately provide reassurance, online anxiety-amplifying distractions and difficulties with interpreting and managing online health information are likely to drive further online searches. For this reason, anxiety-amplifying factors need to be targeted by any effort to decrease or eliminate cyberchondria. To the extent that some associated factors play a role in cyberchondria, especially in the context of hypochondriasis, they should be addressed as well.

\section{Management Implications}

Several "technical" issues can be addressed to decrease the likelihood of developing cyberchondria. This involves the ways in which online health information and results of online health-related searches are presented. For example, a clear and user-friendly presentation of online health information is likely to decrease the misinterpretation and escalation of health anxiety, as is the ranking of the results of online searches based on true probabilities in the relationships between the specific symptoms and diagnoses [3]. Even with these improvements, the risk of cyberchondria cannot be entirely eliminated. Regardless of the manner in which information about serious and life-threatening diseases is conveyed, the unpleasant or potentially fear-eliciting content of such information will always remain. Also, disagreements between different sources of online information cannot be resolved by improving the clarity of that information. In light of this, what therapeutic recommendations can be given to individuals with cyberchondria?

One study suggests that patients with "moderate-severe health anxiety" should avoid using the Internet for health-related information based on a strong association between health anxiety and cyberchondria [21]. A similar recommendation was implicitly made on the grounds that online health-related searches primarily serve the function of reassurance seeking, thereby maintaining hypochondriasis [19]. However, this recommendation is problematic for at least two reasons. First, a suggestion

Cyberchondria that online health information should be avoided carries a message that such information is inherently threatening, which may be more likely to perpetuate cyberchondria than to reduce it. Second, it seems unrealistic to suggest "abstinence" from any innocuous Internet-related activity in an era that is already heavily Internet-reliant and likely to be even more so in the future. That is, people cannot afford to ignore the Internet as the primary source of health-related information.

Instead of promoting the notion that staying away from online health information is salubrious, treatment approaches to cyberchondria should have two main goals: (1) enabling people to use the Internet for health-related purposes without experiencing a heightened health anxiety; (2) reducing the time spent online for health-related purposes so that other activities would not be neglected and functioning could improve. Reaching these goals will be possible if people are exposed to health-related online material in a gradual, controlled fashion, if they learn that online health information is not inherently threatening or overwhelming, if they become confident that they can manage such information and if they can control any urges to make extensive and unnecessary online health-related searches.

Treatment of cyberchondria should be a part of the comprehensive management of individuals with hypochondriasis if cyberchondria is only one aspect of hypochondriasis. In the absence of hypochondriasis, efforts to address the associated, anxiety-amplifying and maintaining factors should still be made. This requires a careful case formulation identifying these factors in every individual, as well as the specific precipitants (e.g., appearance of a new symptom), function that cyberchondria serves (e.g., reassurance seeking) and its consequences (e.g., neglecting family responsibilities as a result of the time spent performing online health-related searches and the associated distress).

Regardless of the specific factors, psychoeducation about cyberchondria seems to be an essential part of any treatment package. This involves improving online health information literacy that focuses on several issues: (1) what the Internet can and cannot do; (2) distinguishing between credible and non-credible sources of online health information; (3) appraising the results of online health searches critically.

First and foremost, individuals with cyberchondria need to learn that the Internet is only a means of obtaining health-related information and that it is not an omnipotent agent that delivers reassurance reliably or has answers for all health-related questions. Also, the Internet

Psychother Psychosom 2017;86:129-133 DOI: $10.1159 / 000465525$ 
is not a tool for self-diagnosis, despite a temptation to use it as such. Individuals with cyberchondria would benefit from learning that accumulating information about one's symptoms as a result of online health searches is not tantamount to establishing a diagnosis that explains those symptoms. A finding that online health-related searches by individuals with high levels of health anxiety are associated with a greater likelihood of visiting a physician [4, $5,12,13]$ suggests that such searches are not sufficient. Consequently, Internet-derived self-diagnosis should not be the aim of online health-related searches, even when online "symptom checkers" with diagnostic algorithms are used.

Knowing which health websites are reliable and trustworthy and which are not is not as simple as it may seem. Attempts to categorise health websites on the basis of their quality have apparently been met with much resistance and there are no clear criteria or guidelines in this area. The usual suggestion to give more weight to government websites, those that clearly state names of the authors and their credentials, websites that have no financial interests and belong to reputable academic, medical or scientific organisations and sources that frequently update their content and provide scientific evidence and references to support their statements or recommendations [22] may be helpful only to a certain extent. This is because some people are generally mistrustful or even resentful of any official institution and may trust more what they find on the websites of support groups run by patients and the corresponding discussion forums and bulletin boards. Mistrust of physicians is certainly an issue for many individuals with hypochondriasis and high levels of health anxiety $[13,23,24]$, while negative expectations of healthcare professionals have been found to drive seeking of health information online [19]. More often than not, people performing online health-related searches end up comparing several sources of health information as they try to get answers to their queries. If there is a discrepancy between these sources, knowing whom to trust becomes a guessing game, which is often the predicament of individuals with cyberchondria. This is why it is important not only to educate such individuals how to distinguish credible from non-credible sources of health information (as much as that is possible), but also address the issue of trust more broadly.

In a complex area such as health and illness, different opinions and disagreements are more a rule than exception, which may be difficult for individuals with cyberchondria to accept. Therefore, they need to be taught to take into account this complexity and the attendant in- congruity between different sources of health information whenever they try to make sense of the results of their online health searches. This endeavour necessitates a capacity to tolerate the resultant increase in uncertainty, but it also fosters critical and realistic thinking. In the end, such thinking leads to better outcomes than jumping to simple, but catastrophic conclusions about one's symptoms.

\section{Outlook for the Future}

Cyberchondria is a fascinating example of how the digital technology may play an important, though not an exclusive role, in causing psychopathological phenomena. It also points to the novel ways in which people with hypochondriasis, a condition known for centuries, can present. Whether these are two essentially different clinical presentations or conditions is unknown. Therefore, a need to better understand the aetiological links and pathways in the relationships between hypochondriasis, health anxiety and online health-related searches cannot be overemphasised.

Research on cyberchondria is in its infancy. Most of the studies thus far have used a cross-sectional design and have been conducted in relatively small, non-clinical samples, raising concerns about their representativeness. Other issues are a lack of agreement about the concept of cyberchondria and problems with the only assessment tool developed thus far, the Cyberchondria Severity Scale [7]. This instrument has been criticised for being too broad, encompassing items that are not necessarily relevant or specific for cyberchondria and giving cyberchondria almost a status of a disorder, with its subscales and dimensions resembling the diagnostic criteria [2]. The Cyberchondria Severity Scale also has some psychometric deficiencies in that its original five-factor structure could not be confirmed, with a suggestion that one of its subscales, mistrust of medical professionals, should be omitted [25-27]. All this calls for the development of a refined assessment tool for cyberchondria and longitudinal, prospective studies conducted in various populations, including clinical samples.

Waiting for a conceptual consensus on cyberchondria and better understanding of it in order to develop treatments for cyberchondria would be irresponsible. There are measures that can be taken to help individuals who are distressed or feel overwhelmed by anxiety when conducting online health-related searches. At present, these measures are not evidence-based, but they can serve as 
the basis for developing treatment approaches that would be tested in randomised, controlled studies. Finally, it is prudent to recommend that all physicians routinely ask patients about their online health-related searches and impact that these searches have on their well-being and help-seeking behaviour.

\section{Disclosure Statement}

The author does not have any conflict of interest to declare.

\section{References}

1 White RW, Horvitz E: Experiences with Web search on medical concerns and self diagnosis; in Proceedings from the American Medical Informatics Association Annual Symposium 2009 (AMIA 2009). Red Hook, Curran Associates, 2009, pp 696-700.

2 Starcevic V, Berle D: Cyberchondria: an old phenomenon in a new guise? in Aboujaoude E, Starcevic V (eds): Mental Health in the Digital Age: Grave Dangers, Great Promise. New York, Oxford University Press, 2015, pp 106117.

3 White RW, Horvitz E: Cyberchondria: studies of the escalation of medical concerns in Web search. ACM Trans Inform Syst 2009;27:article 23.

4 Baumgartner SE, Hartmann T: The role of health anxiety in online health information search. Cyberpsychol Behav Soc Netw 2011; 14:613-618.

5 Muse K, McManus F, Leung C, Meghreblian B, Williams JM: Cyberchondriasis: fact or fiction? A preliminary examination of the relationship between health anxiety and searching for health information on the Internet. J Anxiety Disord 2012;26:189-196.

6 Starcevic V, Berle D: Cyberchondria: towards a better understanding of excessive health-related Internet use. Expert Rev Neurother 2013;13:205-213.

7 McElroy E, Shevlin M: The development and initial validation of the Cyberchondria Severity Scale (CSS). J Anxiety Disord 2014;28: 259-265.

8 Harding KJ, Skritskaya N, Doherty E, Fallon BA: Advances in understanding illness anxiety. Curr Psychiatry Rep 2008;10:311-317.

9 Singh K, Fox JRE, Brown RJ: Health anxiety and Internet use: a thematic analysis. Cyberpsychol J Psychosoc Res Cyberspace 2016;10: article 4 .
10 Te Poel F, Baumgartner SE, Hartmann T, Tanis $\mathrm{M}$ : The curious case of cyberchondria: a longitudinal study on the reciprocal relationship between health anxiety and online health information seeking. J Anxiety Disord 2016; 43:32-40.

11 Fava GA, Cosci F, Sonino N: Current psychosomatic practice. Psychother Psychosom 2017;86:13-30

12 Eastin MS, Guinsler NM: Worried and wired: effects of health anxiety on information-seeking and health care utilization behaviors. Cyberpsychol Behav 2006;9:494-498.

13 Singh K, Brown RJ: Health-related Internet habits and health anxiety in university students. Anxiety Stress Coping 2014;27:542554.

14 Boelen PA, Carleton RN: Intolerance of uncertainty, hypochondriacal concerns, obsessive-compulsive symptoms, and worry. J Nerv Ment Dis 2012;200:208-213.

15 Starcevic V: Relationship between hypochondriasis and obsessive-compulsive personality disorder: close relatives separated by nosological schemes? Am J Psychother 1990;44:340347.

16 Sakai R, Nestoriuc Y, Nolido NV, Barsky AJ: The prevalence of personality disorders in hypochondriasis. J Clin Psychiatry 2010;71:4147.

17 Kellner R: Psychotherapeutic strategies in the treatment of psychophysiologic disorders. Psychother Psychosom 1979;32:91-100.

18 Fava GA, Grandi S, Rafanelli C, Fabbri S, Cazzaro M: Explanatory therapy in hypochondriasis. J Clin Psychiatry 2000;61:317-322.

19 McManus F, Leung C, Muse K, Williams JMG: Understanding "cyberchondria:" an interpretative phenomenological analysis of the purpose, methods and impact of seeking health information online for those with health anxiety. Cognitive Behaviour Therapist 2014;7:e21.
20 Rao P, Skoric MM: Web searching for health: theoretical foundations for analyzing problematic search engine use; in Datta A, Shulman S, Zheng B, Lin SD, Sun A, Lim EP (eds): Social Informatics. Third International Conference, SocInfo 2011, Singapore, October 2011. Proceedings. Lecture Notes in Computer Science (LNCS) 6984. Berlin, SpringerVerlag, 2011, pp 59-66.

21 Doherty-Torstrick ER, Walton KE, Fallon BA: Cyberchondria: parsing health anxiety from online behavior. Psychosomatics 2016; 57:390-400.

22 Reavley NJ, Jorm AF: Psychoeducation and the Internet; in Aboujaoude E, Starcevic V (eds): Mental Health in the Digital Age: Grave Dangers, Great Promise. New York, Oxford University Press, 2015, pp 155-175.

23 Guo Y, Kuroki T, Yamashiro S, Koizumi S: Illness behaviour and patient satisfaction as correlates of self-referral in Japan. Fam Pract 2002;19:326-332.

24 von Scheele C, Nordgren L, Kempi V, Hetta J, Hallborg A: A study of so-called hypochondriasis. Psychother Psychosom 2010;54: 50-56.

25 Fergus TA: The Cyberchondria Severity Scale (CSS): an examination of structure and relations with health anxiety in a community sample. J Anxiety Disord 2014;28:504-510.

26 Norr AM, Allan NP, Boffa JW, Raines AM, Schmidt NB: Validation of the Cyberchondria Severity Scale (CSS): replication and extension with bifactor modeling. J Anxiety Disord 2015;31:58-64.

27 Barke A, Bleichhardt G, Rief W, Doering BK: The Cyberchondria Severity Scale (CSS): German validation and development of a short form. Int J Behav Med 2016;23:595-605. 